\title{
Structure and Dynamics of Surfactant and Hydrocarbon Aggregates on Graphite: A Molecular Dynamics Simulation Study
}

\author{
Maria Sammalkorpi,*,† Athanassios Z. Panagiotopoulos, ${ }^{\ddagger, \S}$ and Mikko Haataja ${ }^{\dagger, \S}$ \\ Department of Mechanical and Aerospace Engineering, Department of Chemical Engineering, and Princeton \\ Institute for the Science and Technology of Materials (PRISM), Princeton University, Princeton \\ New Jersey 08544
}

Received: September 22, 2007; In Final Form: December 7, 2007

\begin{abstract}
We have examined the structure and dynamics of sodium dodecyl sulfate $(\mathrm{SDS})$ and dodecane $\left(\mathrm{C}_{12}\right)$ molecular aggregates at varying surface coverages on the basal plane of graphite via classical molecular dynamics simulations. Our results suggest that graphite-hydrocarbon chain interactions favor specific molecular orientations at the single-molecule level via alignment of the tail along the crystallographic directions. This orientational bias is reduced greatly upon increasing the surface coverage for both molecules due to intermolecular interactions, leading to very weak bias at intermediate surface coverages. Interestingly, for complete monolayers, we find a re-emergent orientational bias. Furthermore, by comparing the SDS behavior with $\mathrm{C}_{12}$, we demonstrate that the charged head group plays a key role in the aggregate structures: SDS molecules display a tendency to form linear file-like aggregates while $\mathrm{C}_{12}$ forms tightly bound planar ones. The observed orientational bias for SDS molecules is in agreement with experimental observations of hemimicelle orientation and provides support for the belief that an initial oriented layer governs the orientation of hemimicellar aggregates.
\end{abstract}

\section{Introduction}

Studies of surfactant adsorption at solid-liquid interfaces have a long history, not least due to its importance in many industrial processes, including corrosion inhibition, dispersion stabilization, detergency, crude oil refining, purification, and lubrication. Understanding self-aggregation and the physical properties of the resulting aggregate structures on surfaces is often key in further improving these important industrial processes, as well as providing fundamental physical insight into general self-assembly processes. Furthermore, surfactant adsorption may provide novel and exciting means to generate templates for self-assembly across several length scales.

Adsorption and the resulting surface structures have been studied by different experimental techniques such as streaming potential method, ${ }^{1}$ calorimetry, ${ }^{2}$ neutron reflection, ${ }^{3-5}$ ellipsometry ${ }^{6-8}$ and fluorescence spectroscopy, ${ }^{9,10}$ and atomic force microscopy (AFM) ${ }^{11-18}$ Of these, AFM provides the most detailed information on the topology of the assembled surfactant structures at molecular scales: Manne et al. first provided evidence of hexadecyltrimethylammonium bromide $\left(\mathrm{C}_{16} \mathrm{TAB}\right)$ organization in parallel stripes at the interface between graphite and aqueous solution. ${ }^{15}$ In subsequent studies, similar morphologies have been observed for various other surfactants and hydrophobic surfaces ${ }^{11,16,18}$ among them sodium dodecyl sulfate (SDS) on graphite. ${ }^{12,19}$ Because the observed stripe spacing was approximately twice the linear size of the surfactant, it has been postulated that a monolayer where the surfactants were placed head-to-head acted as a precursor for the formation of hemi-

* Corresponding author. E-mail: msammalk@princeton.edu. Formerly M. Huhtala.

$\dagger$ Department of Mechanical and Aerospace Engineering.

$\doteqdot$ Department of Chemical Engineering.

$\S$ Princeton Institute for the Science and Technology of Materials (PRISM). micelles; indications of the formation of such monolayers have been observed recently. ${ }^{20,21}$

These experiments have stimulated computational and theoretical studies on the formation kinetics and structure of the aggregates. From a computational perspective, the challenge has been to incorporate accurately the effects of the aqueous detergent solution and a surface. Traditionally, this has been tackled by effectively integrating out, that is, coarse-graining, the surface, detergent, and solvent molecules. Monte Carlo studies $^{22,23}$ employing such models have provided useful information about the morphological transitions in surfactant surface aggregation, whereas coarse-grained molecular dynamics simulations provide support for the hemicylindrical micelle model. ${ }^{24,25}$ Recently, some atomistic or united-atom molecular dynamics simulations with explicit solvent have been carried out. ${ }^{26-28}$ These simulations were performed by initially placing the molecules either in a dense monolayer perpendicular to the surface or in a hemicylindrical form on the surface and following the subsequent evolution of the system in time. Owing to the specific choices of the initial conditions, the outcomes of these simulations may be biased. To probe the unbiased aggregation kinetics, we have carried out simulations of the formation of the initial monolayer starting from initial configurations that are less likely to bias the outcome. Our main observations are threefold. First, the graphite lattice influences the molecular orientation at the single-molecule level for both SDS and dodecane $\left(\mathrm{C}_{12}\right)$. Second, when multiple $\mathrm{SDS}$ or $\mathrm{C}_{12}$ molecules are introduced into the system, the interplay between moleculesurface and intermolecular interactions strongly affects the submonolayer aggregate structure and dynamics. In particular, owing to the stronger attractive intermolecular interactions in the case of $\mathrm{C}_{12}$, the $\mathrm{C}_{12}$ form planar aggregates while the SDS molecules tend to form linear file-like aggregates. Furthermore, the molecules display only a very weak orientational bias in 
this regime. Third, a re-entrant orientational bias emerges at full coverage for both types of molecules.

The rest of this article is organized as follows. In Section 2 we describe the computational model employed, while Section 3 contains the main results of this work. Finally, a discussion of our results can be found in Section 4 .

\section{Methods}

2.1. Computational Model. The Gromacs 3.3 simulation package ${ }^{29-31}$ including Lennard-Jones and Coulombic site-site interactions in addition to bond stretching, angular bending, and improper and proper dihedral interactions with the united atom parametrization $^{32}$ was employed for the molecular dynamics (MD) simulations. The following strategy was implemented. First, the energies of the initial configurations were minimized with the steepest descent method. After the initialization, all simulations were performed in the NVT ensemble using the Berendsen thermostat ${ }^{33}$ with a coupling time constant of 0.1 ps. SDS/C 12 , sodium counterions, and water were thermostated as separate groups. The bond lengths of the detergent and hydrocarbon molecules were constrained by the LINCS algorithm ${ }^{34}$ and those of the water molecules by SETTLE. ${ }^{35}$ A time step of $2 \mathrm{fs}$ was employed in all simulations reported here. The Lennard-Jones interactions were cut off at $1 \mathrm{~nm}$, and the full particle-mesh Ewald method ${ }^{36}$ was employed for the long-ranged electrostatic interactions in order to minimize artifacts resulting from artificially truncating such interactions. ${ }^{37-39}$

We employed an SDS parametrization discussed in detail in ref 40 and available online at www.softsimu.org. The remaining parameters follow the united atom parametrization of ref 32 . The graphite surface was built from fixed Gromacs carbon atoms of type $C B$ placed in a hexagonal pattern with $1.39 \AA$ nearestneighbor distance corresponding to the equilibrium $C B-C B$ distance. The atom type $C B$ was chosen because it represents a bare carbon atom in a 5- or 6-membered ring and thus it was considered to best represent the $\mathrm{sp}^{2}$-hybridized carbon in graphite. Three graphene planes are stacked in ABC-stacking with an interlayer distance of $3.35 \AA$. We verified that the results are not stacking-dependent; that is, ABA-stacking produces the same results. Although a single graphene layer is sufficient to induce the observed orientational behavior at the single-molecule level, three layers were employed to ensure that the behavior of molecular aggregates on the given graphite plane was not affected by molecules adsorbed at the opposing plane. The carbon atoms in the graphene layers were fixed, and periodic boundary conditions were employed to create an infinite graphite surface. Finally, the simple point charge (SPC) model $^{41}$ was adopted for the water molecules. Visualizations of all molecular configurations were done using VMD. ${ }^{42}$

2.2. Simulated System. We studied the behavior of single and multiple (up to a complete monolayer) SDS and $\mathrm{C}_{12}$ molecules on the graphite surface as a function of surface coverage. The SDS or $\mathrm{C}_{12}$ molecules were initially placed in a square lattice with their molecular axis perpendicular to the plane of graphite in bulk water, see Figure 1. For the single-molecule simulations, the graphite slab has dimensions $4.57 \times 5.00 \mathrm{~nm}^{2}$ (912 carbon atoms in each 3 layers) and for the multiple molecule simulations the box is increased to $6.74 \times 7.09 \mathrm{~nm}^{2}$ (1904 carbon atoms in each 3 layers). The simulation box dimension perpendicular to the graphite plane, that is, the combined thickness of the graphite and the water slab, was set as $z=4.5 \mathrm{~nm}$ for the simulations involving a single SDS or a $\mathrm{C}_{12}$ molecule and $z=8.5 \mathrm{~nm}$ for the multiple-molecule simulations. This corresponds to 2533 water molecules (SDS)
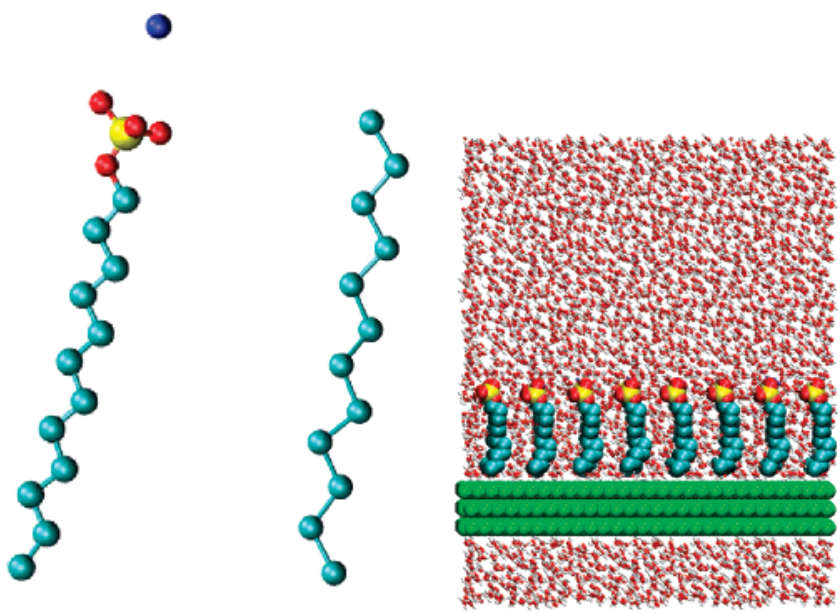

Figure 1. From left to right: sodium dodecyl sulfate and dodecane molecules in the all-trans conformation and an example of an initial configuration. The initial configuration contains 64 SDS molecules in a square lattice of 8 by 8 molecules perpendicular to the three graphene planes. The blue sphere represents the sodium ion, whereas each cyan sphere represents a $\mathrm{CH}_{2}$ or $\mathrm{CH}_{3}$ united-atom group.

or 2540 water molecules $\left(\mathrm{C}_{12}\right)$ in the smaller simulation box and 10739-11761 water molecules in the larger box depending on the number of SDS or $\mathrm{C}_{12}$ molecules. Smaller box dimensions were employed for the single-molecule simulations because 4.5 $\mathrm{nm}$ provides an ample bulk water region for the single molecule not to interact with its own periodic images. However, in the case of multiple molecules (here up to 64), a thicker water slab was required because only a finite fraction of the molecules end up on the surface during the course of the simulation. Indeed, it was ensured that any small aggregates or isolated molecules remaining in the bulk water maintained sufficient distance to the molecules on the surface so as to not directly interfere with the aggregate dynamics and structure on the surface.

With regard to the aggregation kinetics, we observed that after approximately $10 \mathrm{~ns}$ the molecules had either migrated to the graphite surface or formed small metastable micelles in the bulk water. This transient period was regarded as the initial relaxation and was thus not included either in the final analysis or in the interpretation of the simulation results. The remainder of the $50 \mathrm{~ns}$ trajectory formed the basis for the analysis of the results reported here. Furthermore, because we are specifically interested in the behavior of the initial surfactant layer on the graphite surface, the molecules remaining in the bulk water were discarded in the subsequent analysis.

\section{Results}

After initialization, the majority of the SDS and $\mathrm{C}_{12}$ molecules rapidly migrate to the graphite surface within a few nanoseconds and form aggregates. We observed that the fraction of the molecules remaining in the bulk water as small molecular aggregates or individual entities after the relaxation period of $10 \mathrm{~ns}$ corresponds to approximately $15 \%$ of the SDS or $\mathrm{C}_{12}$ molecules in the system. Of course, when the surface monolayer becomes saturated the percentage increases: in the case of 64 SDS molecules, only 45 end up on the surface because 45 adsorbed SDS molecules comprise a complete monolayer on the graphite sheet across our simulation box.

3.1. Aggregate Structure and Dynamics: SDS vs $\mathbf{C}_{\mathbf{1 2}}$. To visualize the behavior of the two molecular systems, Figures 2 and 3 display snapshots of the systems after the initial relaxation period at different surface coverages. Although the $\mathrm{C}_{12}$ mol- 

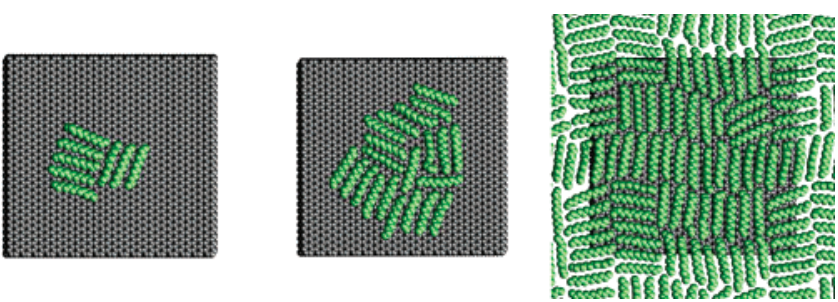

Figure 2. Snapshots of $\mathrm{C}_{12}$ surface configurations at varying surface fillings. The corresponding graphite lattice directions are as in Figure 4.

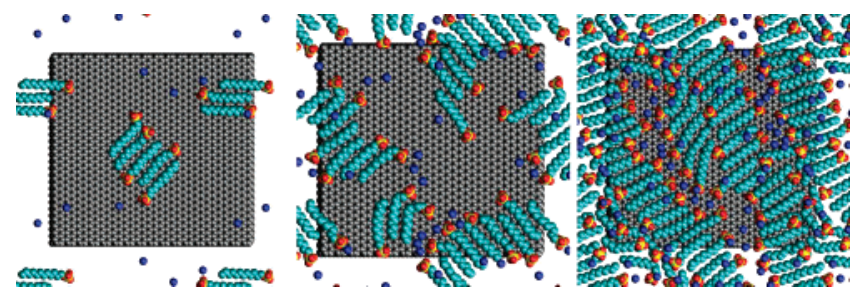

Figure 3. Snapshots of SDS surface configurations at varying surface fillings. The corresponding graphite lattice directions are as in Figure 4.

ecules tend to form compact two-dimensional islands with the $\mathrm{C}_{12}$ molecules in one island (Figure 2), the SDS molecules tend to form much less compact, linear patches (Figure 3). The compact $\mathrm{C}_{12}$ islands rotate in a continuous fashion while migrating on the surface. The SDS patches, in turn, sometimes form single islands only to split into several smaller patches that diffuse individually on the surface. The dynamics of the SDS patches also markedly differ from those of $\mathrm{C}_{12}$ : a linear alignment is preferred, and an SDS patch diffuses preferentially as a single file in a partially filled layer.

Figure 4 presents the distribution of angles made by the vector $C_{i}-C_{i+2}$ in the hydrocarbon backbone, $i$ being the index of the backbone carbon, and a graphite bond, at different surface coverages. This graph reveals three important features. First, both SDS and $\mathrm{C}_{12}$ display a marked orientational bias with respect to the graphite surface at the single-molecule level, the bias being stronger for $\mathrm{C}_{12}$. Second, both $\mathrm{C}_{12}$ and SDS molecules lose most of their orientational preference in the case of partially filled layers. Third, the orientational bias re-emerges for both types of molecules as the monolayer becomes filled.

The explanation for the presence of an orientational bias at the single-molecule level for both $\mathrm{C}_{12}$ and SDS is as follows. Both molecules contain a hydrocarbon tail, which interacts with the surface through the methyl group-graphite interaction. Note that the methyl-methyl equilibrium distance differs from the nearest-neighbor $\mathrm{C}-\mathrm{C}$ bond length in the graphite lattice: in the present model the former is given by $a_{\mathrm{hc}}=1.53 \AA$ while the latter is $a_{\text {gra }}=1.39 \AA$. The methyl-methyl equilibrium distance of the model is in excellent agreement with literature values: the $\mathrm{CH}_{2}-\mathrm{CH}_{2}$ bond in a methyl chain has been reported as $1.524 \AA$, the $\mathrm{CH}_{2}-\mathrm{CH}_{3}$ terminal methyl bond has been reported as $1.513 \AA$, and the overall $\mathrm{C}^{*}-\mathrm{C}^{*}$ bond in organic compounds as $1.53 \AA{ }^{43}$ For the graphite bond, the literature value is $1.42 \AA{ }^{43}$ Although graphite bonds angle at $120^{\circ}$, the hydrocarbon equilibrium angle is somewhat smaller, in the model the angle between $\mathrm{CH}_{2}-\mathrm{CH}_{2}-\mathrm{CH}_{2 / 3}$ bonds is $111^{\circ}$. Taken at face value, these numbers would indicate that the hydrocarbon tails would be incommensurate with the graphite surface regardless of orientation. However, there are two special directions in which some commensurability can be achieved. These correspond to $30^{\circ}$ and $0 / 60^{\circ}$. In the former case, the tail would be fully commensurate if the bond lengths and angles in the hydrocarbon tail and in the surface matched perfectly. In the latter direction, the graphite lattice period is $3 a_{\text {gra. }}$. The hydrocarbon tail zig-zags with a period of $2 \sin \left(55.5^{\circ}\right) a_{\mathrm{hc}}$, which provides a partial commensurability; more precisely, two out of every three methyl groups can obtain a match close to the ideal graphite interlayer atom positioning. Furthermore, the molecule on the surface is free to deform in order to obtain a better match with the graphite lattice. Together, the presence of favorable orientations with regard to the graphite lattice and the ability of the molecules to deform conspire to generate the observed orientational bias.

An isolated $\mathrm{C}_{12}$ orients more strongly than an isolated SDS because of the presence of the hydrophilic head group, which causes the SDS molecule to be less tightly bound to the surface. However, as more molecules are introduced into the system, the interplay between lateral and perpendicular interactions determines the degree of orientational order. Specifically, the head-head repulsion of the SDS molecules makes the SDS molecules much more weakly bound together. As a result of this, $\mathrm{C}_{12}$ forms tightly bound diffusing patches, see Figure 2, while SDS forms linear aggregates. In both cases, intermolecular interactions and fluctuations lead to a marked decrease in the orientational order in the case of partially filled monolayers. As the layer fills up, the effect of fluctuations is reduced greatly and the molecules begin to align along the graphite lattice, displaying a re-emergent orientational bias. Note that although one filled SDS monolayer (49 SDS) exhibits a strong peak at $0 / 60^{\circ}$, the other, that is, 64 SDS, peaks at $30^{\circ}$, as does the filled $\mathrm{C}_{12}$ monolayer $\left(64 \mathrm{C}_{12}\right.$ ). We believe that this is due to both orientations $\left(0 / 60\right.$ and $30^{\circ}$, respectively) being local energy minima as discussed above. Additionally, the rotational motion of the molecules becomes very sluggish as the layer fills up because it requires collective and concerted motion of the molecules occurring at longer time scales than those simulated here (50 ns).

To further examine the behavior of adsorbed molecules on the graphite surface, we computed the lateral distance distributions of the methyl groups with respect to the underlying graphite lattice. Additionally, because the only difference between the molecules is the head group (and the counterions), we also examined the distance distributions of the SDS head group sulfur atoms with respect to the underlying graphite lattice. The data are shown in Figure 5. The methyl groups in the case of an isolated $\mathrm{C}_{12}$ molecule have a strongly nonuniform distribution, and some preference is also observed for the single SDS molecule. Contrast this to the cases of multiple $\mathrm{C}_{12}$ or SDS molecules, where the methyl groups do not appear to exhibit any preferential positioning with respect to the underlying graphite. Interestingly, the sulfur atom of an isolated SDS molecule on graphite has a preferential positioning with respect to underlying graphite lattice; furthermore, this preference also persists in the presence of multiple SDS molecules, see Figure 5.

Let us now focus on the underlying reason for the preferred position of the SDS head group on graphite. There are two plausible scenarios. First, the head group position could be fully induced by the tail, which becomes more or less commensurate with the lattice. Second, the direct interaction of the head group with the lattice could contribute to the bias. To test the latter scenario, we carried out the following procedure. First, we picked a relaxed $10 \mathrm{~ns}$ configuration of a 9 SDS system. Then, the methyl tails were removed and the resulting voids were filled with water molecules, thus leaving bare $\mathrm{SO}_{4}{ }^{-}$groups and $\mathrm{Na}^{+}$ ions in aqueous solution. The partial charge of the $\mathrm{S}$ atom was 

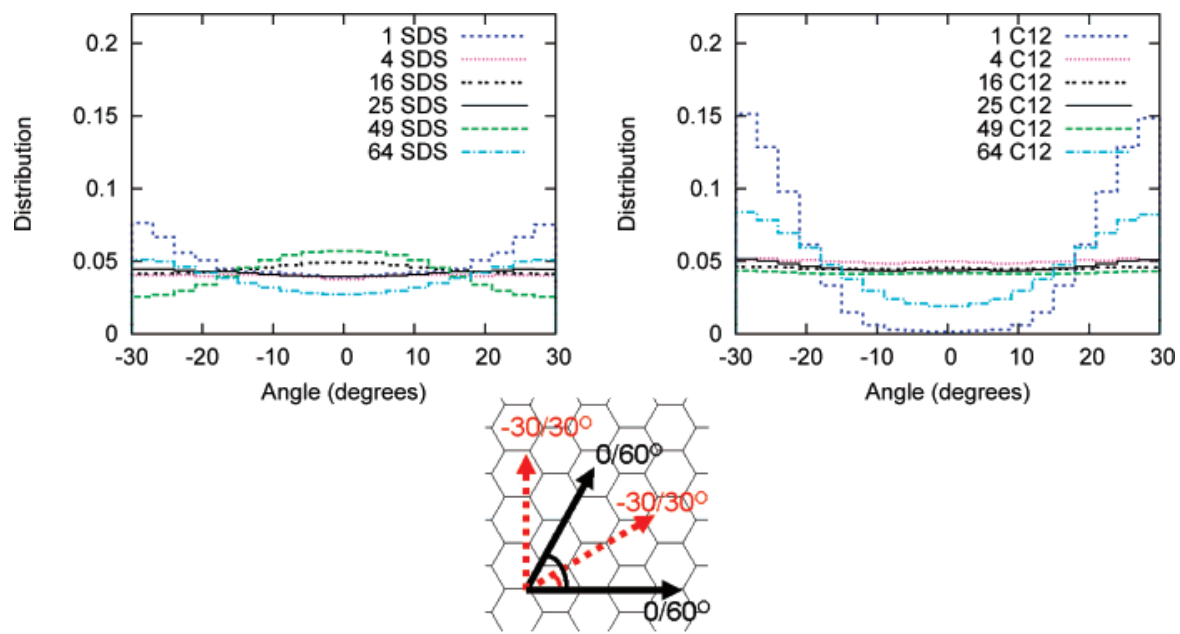

Figure 4. SDS and $\mathrm{C}_{12}$ orientation with respect to graphite surface. The angle is calculated as the angle between the vector formed by hydrocarbon backbone atoms $i$ and $i+2$ and the graphite bond. Because graphite bonds are oriented at $120^{\circ}$ intervals, we obtain a distribution between $-30^{\circ}$ and $30^{\circ}$. The distribution is normalized such that at random distribution each of the 20 bins has a value of 0.05 equalling 1.0 over the whole interval.
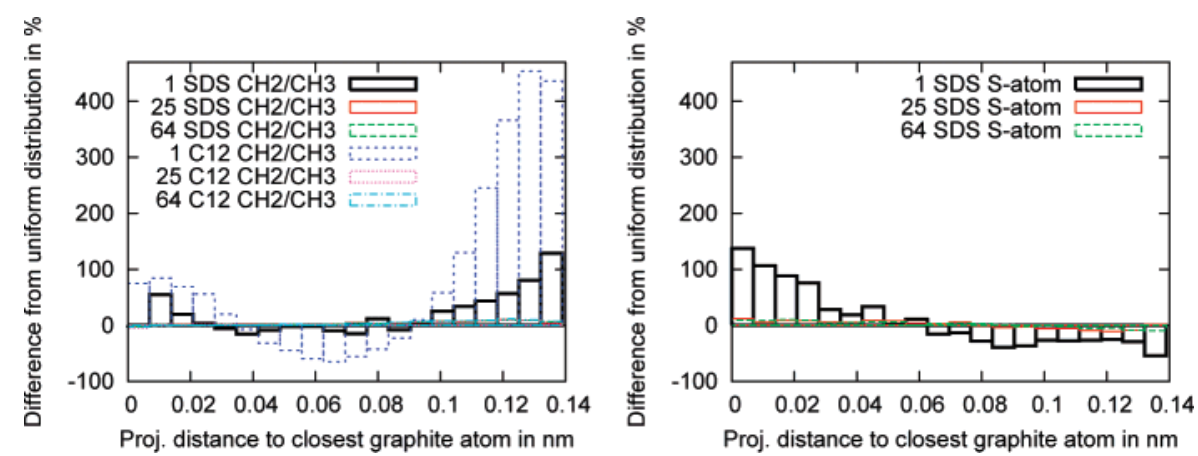

Figure 5. SDS and $\mathrm{C}_{12}$ methyl group and SDS head group (S-atom) lateral distance distribution difference to uniform distribution in percents.

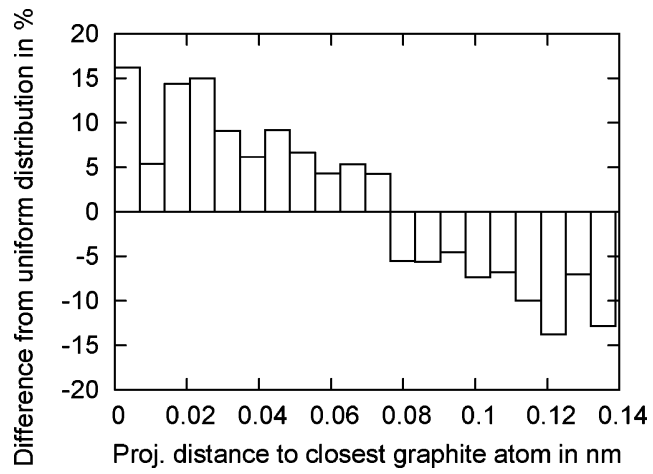

Figure 6. $\mathrm{SO}_{4}^{-}$group (SDS head group without the tail) lateral distance distribution to closest graphite atom. The distribution is presented as the percentage difference from uniform distribution.

kept untouched, and the remaining charge was divided equally between the four oxygen atoms. All other parameters were retained. The $\mathrm{SO}_{4}{ }^{-}$groups were fixed in the direction perpendicular to the graphite surface at the position they were in the original SDS trajectory frame to prevent the hydrophilic $\mathrm{SO}_{4}{ }^{-}$ groups from leaving the surface yet allowing for unhindered mobility in the graphite plane. The simulation was then run for an additional $5 \mathrm{~ns}$, and the position distribution was computed. The data for this constrained simulation is presented in Figure 6. The results show that although the hydrocarbon tail may be required to bind the solvated molecule to a hydrophobic surface the head group alone prefers certain locations along the graphite plane and thus contributes to the observed positional bias.

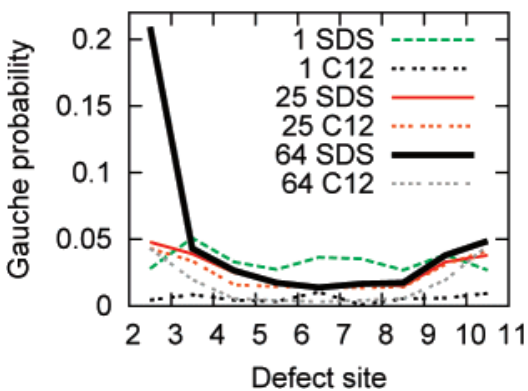

Figure 7. Gauche defect probability.

3.2. Molecular Conformations and Nearest-Neighbor Orientation Correlations. To further characterize the molecular conformations on the surface, we computed the gauche defect probability of the molecules on the surface at several different coverages. The data is shown in Figure 7. As expected, SDS has a higher defect probability than the corresponding $C_{12}$ system because of the hydrophilic head group. Altogether the defect probabilities are very small, with the exception of the head groups of the 64 SDS molecule system. Specifically, the data indicates that in this case approximately $20 \%$ of the SDS molecules have their head group tilted away from the surface as the monolayer becomes filled. This is not surprising: as the monolayer fills up, it becomes energetically favorable to maximize the number of tail-graphite contacts at the expense of promoting kinks that force some of the head groups away from the surface. To put the magnitude of the defect probabilities in perspective, the corresponding data for SDS micelles and a single SDS molecule in bulk water is plotted in Figure 8. The 


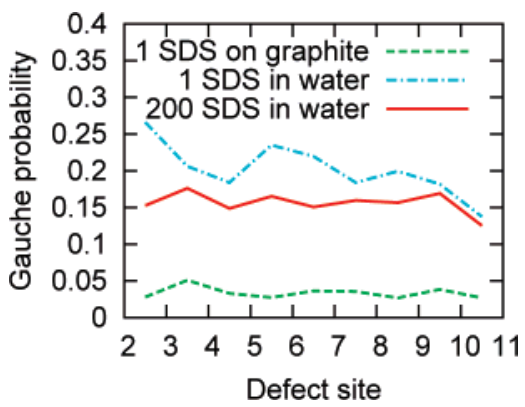

Figure 8. Comparison of gauche defect probability of SDS in bulk water as an individual molecule (1 SDS), in micelles (200 SDS molecules), and on graphite (1 SDS). The description of SDS simulations in bulk water is provided in ref 40 .

details of the SDS micelle and single-molecule simulations in bulk water have been discussed in ref 40 . The data clearly shows that the presence of the graphite surface suppresses the formation of gauche defects dramatically. Naturally, this is a direct consequence of the fact that the molecules are bound to the surface by hydrophobic interactions, and hence the gauche defect formation normal to the surface is suppressed.

With regard to the packing of individual molecules in the aggregates, the intuitive way to pack the SDS molecules in a single file is in an antiparallel fashion so as to minimize the repulsive interactions between the head groups. We have quantified this tendency by computing the orientation angle distribution between nearest-neighbor molecules. Indeed, in partially filled monolayers the SDS molecules prefer the antiparallel orientation. However, as the monolayer fills up, a visual inspection of configurations in Figure 3 shows indications of more parallel packing. The corresponding data is presented in Figure 9, which shows that the fraction of parallel nearestneighbor SDS molecules increases as the monolayer fills up. As expected, the $\mathrm{C}_{12}$ results in a symmetric molecule-molecule orientation distribution. The $90^{\circ}$ peak of $64 \mathrm{C}_{12}$ molecules corresponds to the square tile packing observed as the monolayer fills, see Figure 2.

\section{Discussion}

We have demonstrated here that graphite $-\mathrm{SDS} / \mathrm{C}_{12}$ interactions induce a molecular orientation bias at the single adsorbed molecule level. Surprisingly, this bias disappears at nonzero surface coverages only to re-emerge at full coverage. We propose that this orientational bias is responsible for the observed orientational bias of hemimicellar CTAC aggregates as reported in ref 44 . Furthermore, we have shown that the fraction of parallel nearest-neighbor SDS molecules increases as the monolayer fills, which further supports the existing theories of hemimicelle formation on two files of surfactant molecules oriented in parallel. We have also demonstrated that

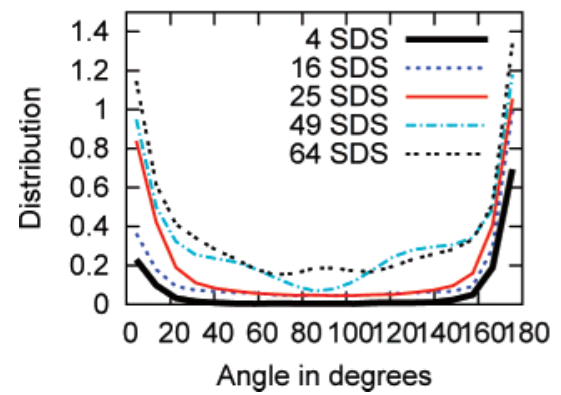

the graphite surface suppresses the configurational defect probabilities drastically in the molecules adsorbed on the surface in comparison with molecules in bulk water or in micellar aggregates. This is an important observation because such low defect densities allow for the formation of straight files with a high degree of local order, which is required for the formation of micrometer-long straight hemimicellar aggregates observed in the experiments.

The observation of the single-molecule orientational bias deserves further comments, especially in the light of analysis in refs 44 and 45 wherein it was argued that collective behavior is required for the appearance of the orientational bias. This argument was based on the calculation of the adsorption energy of a rigid surfactant molecule at varying orientations on graphite. Specifically, the calculation indicated that the energy difference between the $0 / 60^{\circ}$ and $30^{\circ}$ orientations was comparable to the thermal energy, and therefore only a weak bias was predicted, in contrast with our results. To explain this discrepancy, we optimized the geometry of a single SDS molecule by energy minimization of the structure in bulk water within the employed GROMACS ensemble and studied the energetics when this (now rigid) molecule was translated stepwise on the graphite surface. In agreement with ref 44 , we observe that the energy difference between the orientations corresponding to $0 / 60^{\circ}$ and $30^{\circ}$ orientation for this artificially rigid SDS molecule is comparable to the thermal energy. However, we argue that this energy difference is sufficient to bias one of the orientations over another, as can be seen in Figure 4. A further contribution is obtained because in the full MD simulations the molecules are able to deform and adjust to the local structure of the surface. It is precisely this degree of freedom that facilitates the observed orientational bias for the single SDS (or $\mathrm{C}_{12}$ ) molecule. Nevertheless, our results imply that the orientational bias disappears at low coverages only to re-emerge at complete filling. Therefore, our results agree with those in refs 44 and 45 , namely, that collective behavior is at the root of the observed hemimicellar orientational bias in experiments.

Beyond the orientational bias, we have also reported that both the SDS and the $\mathrm{C}_{12}$ molecules prefer patch-like initial formations that diffuse on the surface. The patch formation is stronger in $\mathrm{C}_{12}$ than in SDS aggregates, for which the head-head repulsion leads to the formation of linear file-like domains. Our results demonstrate that the charged head group of SDS also strongly interacts with the graphite surface.

Although antiparallel orientation is favored for low SDS surface coverage, we observe an increased fraction of parallel oriented SDS molecules at elevated surface coverage. This observation supports the theory that the experimentally observed hemimicellar aggregates form on a head-to-head oriented files of parallel-oriented SDS molecules. Unfortunately, observing the full transition to ordered two-column structures is not

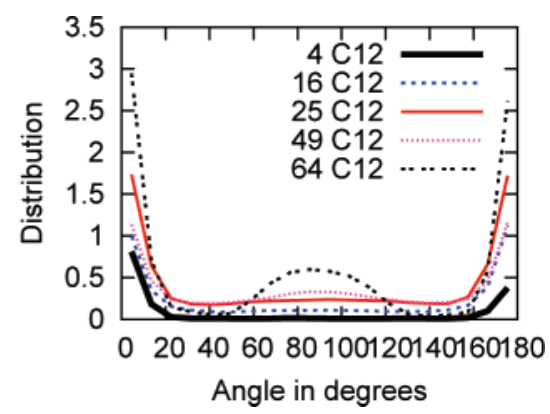

Figure 9. Intermolecular orientation calculated between neighboring molecules for SDS and $\mathrm{C}_{12}$ molecules. $0^{\circ}$ indicates parallel orientation, whereas $180^{\circ}$ indicates antiparallel orientation. The distributions have been scaled by the number of molecules on the surface. 
something that can be investigated at the moment using MD simulations because of time scale issues. It may also be that the transition does not actually take place in a monolayer but additional adsorbed layers are required to induce a two-column orientation. For SDS solvated in water, the formation of oriented hemimicelles on the graphite surface has been reported, ${ }^{12,19}$ but for $C_{12}$ we are not aware of publications in which the $C_{12}$ are in contact with the surface in the presence of water. In the absence of water, however, $\mathrm{C}_{12}$ and related alkanes have been reported to form ordered monolayers experimentally ${ }^{46-49}$ and theoretically. ${ }^{50-54} \mathrm{We}$ would like to point out that the presence of water changes the phase behavior of the system, and therefore these results cannot be compared directly with the ones reported in this work.

The simulation results presented here arise from a delicate interplay between the head group repulsion and tail-tail attraction, which raises the question of how sensitive the observations are to the employed model and choice of parametrization. The repulsive SDS head group interactions are dominated by the electrostatic interactions. This implies that the partial charges of the head group atoms together with the local screening due to the sodium counterions affect the strength of these repulsive interactions. The parametrization employed in this work has been shown to reproduce counterion binding within experimental measurement accuracy for SDS micelles. ${ }^{40}$ With regard to the tails, they are uncharged, like the surface, and therefore the tail-tail attraction and surface-tail attraction are dictated by the van der Waals interactions. These are described by the standard $(6,12)$-Lennard-Jones interaction in which the strength of the interaction is atom-type-dependent. Within the Gromacs force field parametrization, all of the somewhat similarly bonded carbon atoms as the chosen $\mathrm{CB}$ share the same $(6,12)$-parameters; likewise, OM and OS, which are used in the head group, have identical $(6,12)$-parameters. However, the interactions describing van der Waals interactions in different classical force fields have been parametrized to reproduce different thermodynamic quantities. Because different frames of reference are used, the van der Waals interactions for a certain atom type do vary between different force fields. ${ }^{55,56}$

To assess the potential used in this work, we calculated the graphite interplanar interaction energy per surface atom at $T=$ $0 \mathrm{~K}$ in vacuum, and obtained a value of $40 \mathrm{meV} /$ atom with our parametrization. This value compares well with those reported from experiments, namely, $43,{ }^{57} 35,{ }^{58}$ and $52 \mathrm{meV} /$ atom, ${ }^{59}$ whereas other theoretical calculations range typically between 20 and $60 \mathrm{meV} /$ atom $^{60-66}$ It is noteworthy that theoretical values as low as an order of magnitude smaller or as high as $170 \mathrm{meV} /$ atom have also been reported. For a single hydrocarbon molecule, here $\mathrm{C}_{12}$ on graphite, the model-predicted adsorption energy at $T=0 \mathrm{~K}$ is $1.3 \mathrm{eV}$ in vacuum. Although reasonable, this quantity is considerably less straightforward to compare with pre-existing data because its precise value is renormalized by both a finite temperature and the presence of solvent. Although the choice of the force field parametrization may affect, for example, the shape and sharpness of the molecular orientation distributions, we believe that the presence of the orientational bias is a robust phenomenon that would be observed with most physically reasonable force field parametrizations. Of course, the strength of the bias may vary between different parametrizations because the relative magnitudes of van der Waals attraction between the tails and the tails and the surface are different. Another factor contributing to the bias for SDS is the relative magnitude between the screened head-head repulsion in comparison to the van der Waals forces, whose balance depends on both temperature and ionic strength of the solution.

In conclusion, we have demonstrated that surfactants and surfactant aggregates orient on the graphite surface along crystallographic directions. These observations provide molecular-level information about the origins of the hemimicelle aggregation on graphite, which can be employed to tailor the properties of surfactant aggregates on surfaces.

Acknowledgment. M.S. thanks Antti-Pekka Hynninen for scientific discussions and help with running the simulations. This work has been supported by Academy of Finland (M.S.), through an NSF-DMR Grant No. 0449184 (M.H.) and NSFMRSEC Program, Grant No. DMR-0213706 at Princeton University.

\section{References and Notes}

(1) Fuerstenau, D. W. J. Phys. Chem. 1956, 60, 981-985.

(2) Király, Z.; Findenegg, G. H. J. Phys. Chem. B 1998, 102, 12031211.

(3) McDermott, D. C.; McCarney, J.; Thomas, R. K.; Rennie, A. R. J. Colloid Interface Sci. 1994, 162, 304-310.

(4) Penfold, J.; Thomas, R. K. J. Phys.: Condens. Matter 1990, 2, $1369-1412$

(5) Penfold, J.; Staples, E. J.; Tucker, I.; Thompson, L. J. Langmuir 1997, 13, 6638-6643.

(6) Tiberg, F.; Joensson, B.; Tang, J.; Lindman, B. Langmuir 1994, 10, 2294-2300 3722 .

(7) Tiberg, F.; Joensson, B.; Lindman, B. Langmuir 1994, 10, 37140.

(8) Luciani, L.; Denoyel, R. J. Colloid Interface Sci. 1997, 188, 75-

(9) Levitz, P.; van Damme, H.; Keravis, D. J. Phys. Chem. 1984, 88, 2228-2235.

(10) Chandara, P.; Somasundaranan, P.; Turro, N. J. J. Colloid Interface Sci. 1987, 117, 31-46.

(11) Manne, S.; Gaub, H. E. Science 1995, 270, 1480-1482.

(12) Wanless, E. J.; Ducker, W. A. J. Phys. Chem. 1996, 100, 32073214.

(13) Ducker, W. A.; Grant, L. M. J. Phys. Chem. 1996, 100, 1150711511

(14) Ducker, W. A.; Wanless, E. J. Langmuir 1996, 12, 5915-5920.

(15) Manne, S.; Cleveland, J. P.; Gaub, H. E.; Stucky, G. D.; Hansma,

P. K. Langmuir 1994, 10, 4409-4413.

(16) Patrick, H. N.; Warr, G. G.; Manne, S.; Aksay, I. A. Langmuir 1997, 13, 4349-4356.

(17) Patrick, H.; Warr, G.; Manne, S.; Aksay, I. Langmuir 1999, 15 (5), 1685-1692.

(18) Jaschke, M.; Butt, H.-J.; Gaub, H. E.; Manne, S. Langmuir 1997, 13, 1381-1384.

(19) Ducker, W. A.; Wanless, E. J. J. Phys. Chem. 1996, 100, 3207 -3214 .

(20) Kawasaki, H.; Ban, K.; Maeda, H. J. Phys. Chem. B 2004, 108, 16746-16752.

(21) Király, Z.; Findenegg, G. H. Langmuir 2005, 21, 5047-5054.

(22) Wijmans, C.; Linse, P. J. Phys. Chem. 1996, 100, 12583-12591.

(23) Zheng, F.; Zhang, X.; Wang, W.; Dong, W. Langmuir 2006, 22, 11214-11223.

(24) Srinivas, G.; Nielsen, S. O.; Moore, P. B.; Klein, M. L. J. Am. Chem. Soc. 2006, 128, 848-853.

(25) Bandyopadhyay, S.; Shelley, J.; Tarek, M.; Moore, P.; Klein, M. J. Phys. Chem. B 1998, 102, 6318-6322.

(26) Shah, K.; Chiu, P.; Jain, M.; Fortes, J.; Moudgil, B.; Sinnott, S. Langmuir 2005, 21, 5337-5342.

(27) Shah, K.; Chiu, P.; Sinnott, S. B. J. Colloid Interface Sci. 2006, 296, 342-349.

(28) Dominguez, H. J. Phys. Chem. B 2007, 111, 4054-4059.

(29) Berendsen, H. J. C.; van der Spoel, D.; van Drunen, R. Comput. Phys. Commun. 1995, 91, 43-56.

(30) Lindahl, E.; Hess, B.; van der Spoel, D. J. Mol. Model. 2001, 7, 306-317.

(31) van der Spoel, D.; Lindahl, E.; Hess, B.; Groenhof, G.; Mark, A. E.; Berendsen, H. J. C. J. Comput. Chem. 2005, 26, 1701-1718.

(32) Berger, O.; Edholm, O.; Jahnig, F. Biophys. J. 1997, 72, 20022013.

(33) Berendsen, H. J. C.; Postma, J. P. M.; van Gunsteren, W. F.; DiNola, A.; Haak, J. R. J. Chem. Phys. 1984, 81, 3684-3690. 
(34) Hess, B.; Bekker, H.; Berendsen, H. J. C.; Fraaije, J. G. E. M. J. Comput. Chem. 1997, 18, 1463-1472. 962.

(35) Miyamoto, S.; Kollman, P. A. J. Comput. Chem. 1992, 13, 952-

(36) Essman, U.; Perela, L.; Berkowitz, M. L.; Darden, T.; Lee, H.; Pedersen, L. G. J. Chem. Phys. 1995, 103, 8577-8592.

(37) York, D. M.; Darden, T. A.; Pedersen, L. G. J. Chem. Phys. 1993, 99, 8345-8348.

(38) Patra, M.; Karttunen, M.; Hyvönen, M. T.; Lindqvist, P.; Falck, E.; Vattulainen, I. Biophys. J. 2003, 84, 3636-3645.

(39) Patra, M.; Karttunen, M.; Hyvönen, M. T.; Falck, E.; Vattulainen, I. J. Phys. Chem. B 2004, 108, 4485-4494.

(40) Sammalkorpi, M.; Karttunen, M.; Haataja, M. J. Phys. Chem. B 2007, 111, 11722-11733.

(41) Berendsen, H. J. C.; Postma, J. P. M.; van Gunsteren, W. F.; Hermans, J. In Intermolecular Forces; Pullman, B., Ed.; Reidel: Dordrecht, 1981; pp 331-342.

(42) Humphrey, W.; Dalke, A.; Schulten, K. J. Mol. Graphics 1996, 14, 33-38.

(43) Lide, D. R., Ed. CRC Handbook of Chemistry and Physics, 88th ed.; CRC Press: Boca Raton, FL, 2007.

(44) Saville, D. A.; Chun, J.; Li, J.-L.; Schniepp, H. C.; Car, R.; Aksay, I. A. Phys. Rev. Lett. 2006, 96, 018301.

(45) Chun, J.; Li, J.-L.; Car, R.; Aksay, I.; Saville, D. J. Phys. Chem. B 2006, 110, 16624-16632.

(46) Rabe, J.; Buchholz, S. Science 1991, 253, 424-427.

(47) Wawkuschewski, A.; Cantow, H.-J.; Magonov, S. N. Langmuir 1993, 9, 2778-2781.

(48) Clarke, S. M.; Inaba, A.; Arnold, T.; Thomas, R. K. J. Therm. Anal. Calorim. 1999, 57, 643-651.
(49) Arnold, T.; Thomas, R. K.; Castro, M. A.; Clarke, S. M.; Messe, L.; Inaba, A. Phys. Chem. Chem. Phys. 2002, 4, 345-351.

(50) Hentschke, R.; Schürmann, B. L.; Rabe, J. P. J. Chem. Phys 1992, $96,6213-6221$

(51) Hentschke, R.; Winkler, R. G. J. Chem. Phys 1993, 99, 55285534

(52) Pint, C. L. Surf. Sci. 2006, 600, 921-932.

(53) Pint, C. L.; Roth, M. W.; Wexler, C. Phys. Rev. B 2006, 72, 085422.

(54) Yang, H.; Li, Z.-S.; Lu, Z.-Y.; Sun, C.-C. Surf. Sci. 2006, 600, $1213-1220$

(55) A. D. M., Jr. J. Comput. Chem 2004, 25, 1584-1604.

(56) Yin, D.; A. D. M., Jr. J. Comput. Chem. 1998, 19, 334-348.

(57) Girifalco, L. A.; Law, R. A. J. Chem. Phys. 1956, 25, 693-697.

(58) X. 'Benedict, L.; Chopra, N. G.; Cohen, M. L.; Zettl, A.; Louie, S. G.; Crespi, V. H. Chem. Phys. Lett. 1998, 286, 490-496.

(59) Zacharia, R.; Ulbricht, H.; Hertel, T. Phys. Rev. B 2004, 69, 155406.

(60) Brennan, R. O. J. Chem. Phys. 1952, 20, 40-48.

(61) Schabel, M. C.; Martins, J. L. Phys. Rev. B 1992, 46, 7185-7188.

(62) Trickey, S. B.; Müller-Plathe, F.; Diercksen, G. H. F. Phys. Rev. B 1992, 45, 4460-4468.

(63) Charlier, J.-C.; Gonze, X.; Michenaud, J. P. Europhys. Lett. 1994 $28,403-408$.

(64) Hasegawa, M.; Nishidate, K.; Iyetomi, H. Phys. Rev. B 2007, 76, 115424.

(65) Girifalco, L. A.; Hodak, M. Phys. Rev. B 2002, 65, 125404.

(66) Rydberg, H.; Jacobson, N.; Hyldgaard, P.; Simak, S. I.; Lundqvist, B. I.; Langreth, D. C. Surf. Sci. 2003, 532-535, 606-610. 